\title{
LA PERCEPCIÓN DE LA COMUNIDAD ACADÉMICA SOBRE EL SISTEMA DE INVESTIGACIÓN YEVALUACIÓN EN COMUNICACIÓN
}

\section{ACADEMIC'S PERCEPTION ABOUT THE RESEARCH AND EVALUATION SYSTEM IN COMMUNICATION}

\author{
Ramón Martínez García \\ | ramon.martinez@uma.es | \\ Universidad de Málaga
}

Resumen: La finalidad de este trabajo es analizar el contexto en el que se desarrolla la Comunicación como práctica social desde una dimensión discursiva. Este trabajo parte de una perspectiva cualitativa de naturaleza exploratoria, por lo que carece de hipótesis iniciales. Su objetivo es extraer las líneas de opinión predominantes de la comunidad académica sobre el Sistema de Investigación y Evaluación en Comunicación y su influencia en el contexto académico y científico. Para ello se ha lanzado una encuesta online tipo Likert sobre un censo de 2.418 investigadores en Comunicación en España, alcanzando 604 respuestas, lo que supone una tasa de respuesta del 24,97 por ciento. Se asume que la comunidad investigadora forma parte de un mismo ecosistema académico y que todos los sujetos se encuentran estrechamente interrelacionados entre sí. Por ello, el diseño de la encuesta obedece a un modelo basado en tres dimensiones: la producción de conocimiento, la gestión de instituciones y la evaluación de la investigación. Entre las principales conclusiones destaca la percepción generalizada de que el sistema de investigación y evaluación ha provocado una dinámica funcionalista que condiciona la forma de investigar, afectando de lleno a la innovación y disrupción del discurso científico. Palabras clave: metainvestigación en comunicación; discurso científico; sistemas de evaluación; estudios del contexto; encuesta tipo Likert. 


\begin{abstract}
This paper aims to analyze the context in which the Communication is developed as a social practice from a discursive dimension. This work is based on a qualitative perspective of an exploratory nature, so it does not have initial hypotheses. Its objective is to extract the academic community predominant opinion lines on the Communication Research and Evaluation System and its influence in the academic and scientific context. To this end, a Likert-type online survey has been launched on a census of 2,418 researchers in Communication in Spain, reaching 604 responses, representing a response rate of $24.97 \%$. It is assumed that the research community is part of the same academic ecosystem, being all subjects closely interrelated. Therefore, the design of the survey obeys to a model based on three dimensions: knowledge production, institution management and research evaluation. Among the main conclusions stands out the generalized perception that the research and evaluation system has provoked a functionalist dynamic. It conditions the form of research, fully affecting innovation and interruption of scientific discourse. Keywords: Communication meta-research; scientific discourse; evaluation systems; context studies; Likert scale survey.
\end{abstract}

\title{
1. Introducción
}

En el año 1972, pocos meses después de la aprobación del decreto por el que se constituían las Facultades de Ciencias de la Información' ${ }^{1}$, el profesor Vidal Beneyto publicaba un pequeño libro, ya histórico, en el que proponía un plan alternativo a la organización de la docencia y la investigación en Comunicación en España. Decía el profesor que «en el análisis de la realidad social uno de los fenómenos que habían constituido más unanimidad era la consideración de que la universidad estaba en crisis» (1972: 1).

Para sostener tal afirmación, se apoyaba en lo que denominaba «arbitrismo genesíaco» que ha caracterizado la institucionalización de la universidad en España, en la «confortadora convención social de hacer como si la universidad efectivamente funcionase, cuando todos sabían que no funcionaba» y sobre la Comunicación, en concreto, afirmaba que «la manera cómo se ha organizado el aprendizaje de estas disciplinas sociales es absolutamente incongruente con los supuestos que lo reclaman y lo justifican» (Vidal Beneyto, 1972: 13-14).

El presente estudio busca volver a cuestionar esa realidad, a partir del análisis del contexto en el que se desarrolla la Comunicación como práctica social desde una dimensión discursiva. Este trabajo parte de una perspectiva cualitativa de naturaleza exploratoria, por lo que carece de hipótesis iniciales.

[01] Decreto 2070/1971, de 13 de agosto, por el que se regulan los estudios de Periodismo y demás medios de comunicación social en la Universidad. Ver en https://www.boe.es/boe dias/1971/09/14/pdfs/A14944-14945.pdf 
Su objetivo es extraer las líneas de opinión predominante de la comunidad académica sobre el Sistema de Investigación y Evaluación en Comunicación (a partir de ahora SIEC) y su influencia en el contexto académico y científico. La técnica de investigación empleada es una encuesta online estructurada en tres niveles, interrelacionados entre sí: la producción de conocimiento, la gestión de instituciones y la evaluación de la investigación.

Hasta ahora ha sido muy prolífica la bibliografía que recuenta y mide la producción científica basada en todo tipo de signos externos e indicadores de producción científica. Sin ánimo de establecer una lista numerus clausus, algunos autores que han seguido esa línea son: Jones (1998), DelgadoLópez y Repiso-Caballero (2013), De-Filipo (2013), Caffarel, Ortega y Gaitán (2017), Costa-Sánchez (2017), Martínez y Saperas (2011). Otros autores se han centrado en explicar por qué y cómo investigamos: Caffarel, Domínguez y Romano (1989), Martínez Nicolás (2006), Goyanes y Rodríguez-Gómez (2018), Rodríguez, Goyanes y Rosique (2018), Piñuel y Morales (2018) y Martínez-Nicolás, Saperas, Carrasco-Campos, (2019).

Mientras que es más escasa la línea que incide en una dimensión cualitativa e interpretativa del contexto en comunicación. En este sentido Caffarel (1993), Díaz Nosty (2011), Peñafiel, Torres e Izquierdo (2017), Gaitán, Caffarel, Lozano y Piñuel (2018) y Piñuel y Morales (2018). Este trabajo podría enmarcarse en esta última línea de investigación, aquella que pretende contribuir a completar ese esfuerzo de describir, analizar y reinterpretar el contexto de la Investigación en Comunicación.

\section{Marco teórico. Fundamentos epistemológicos}

En línea con el concepto introducido por Kuhn (1962) de comunidad científica, los factores del contexto que delimitan e influyen sobre un campo particular son múltiples y complejos. En relación con la metainvestigación en Comunicación, el análisis de estos factores debería incluir, además de aspectos históricos (Martínez, Saperas y Carrasco-Campos, 2018) o indicadores objetivos de producción científica (Castillo y Carretón, 2010), otros elementos que influyen en el desarrollo de una práctica social determinada.

El análisis del contexto «ayuda a comprender por qué la investigación que se realiza en un cierto momento adquiere unas u otras características» (Martínez Nicolás, 2006: 140). Sin embargo, aún no existe una teoría sobre el contexto que sea capaz de aunar ese esfuerzo investigativo, admitiéndose que ésta deba ser construida necesariamente desde una perspectiva transdisciplinar (Van Dijk, 2001: 70). Esto quiere decir que para entender cómo influye el contexto en un determinado fenómeno hay que atender a un modelo plural de 
análisis: diferentes disciplinas, campos y objetos de estudios, diseñados con la misma finalidad, que es entender una determinada realidad. En este caso, analizar una parte del contexto - la percepción de los sujetos - en el que se desarrolla el Sistema de Investigación y Evaluación en Comunicación.

La presente investigación se construye desde un enfoque epistemológico sociocrítico (Denzin, 1970) y pretende ofrecer herramientas de análisis a la comunidad académica que permitan, no sólo reinterpretar el fenómeno, sino participar activa y críticamente en la construcción de otros modelos científicos que cuestionen los enfoques basados en la evidencia para la práctica del conocimiento, que pueden verse como expresión del conservadurismo científico contemporáneo. Este rol activo de la investigación cualitativa se conoce como octavo momento «y se preocupa del avance del discurso moral y el desarrollo de textualidades sacralizadas y aspira a que las ciencias sociales recuperen su lugar de encuentro para la realización de conversaciones críticas acerca de la democracia, raza, género, clase, naciones-estado, globalización, libertad y comunidad» (Denzin y Lincoln, 2005: 1115-1127).

Aunque la práctica de la investigación es ante todo un fenómeno complejo e individual, el enfoque cualitativo proporciona una amplia gama de herramientas, esclarecimiento ${ }^{2} \mathrm{y}$, finalmente, cambio de la práctica misma (Higss, Horsfall y Grace, 2009: 11). Además de este enfoque teleológico, se entiende la realidad como especie de entelequia aristotélica ${ }^{3}$ que por definición es compleja (Morin, 1994) y con una dimensión profundamente discursiva (Wittgenstein, 1953).

Siguiendo esta línea epistemológica, interesa más analizar «por qué la cuestión está en este estado, que el estado de la cuestión en sí» (Martínez Nicolás, 2006: 166). Conviene señalar, no obstante, algunos rasgos que han caracterizado la institucionalización de la Comunicación como campo de conocimiento a punto de cumplirse medio siglo desde su nacimiento en España. Entre los elementos que han descrito el devenir histórico de este campo destacan el arranque tardío debido al férreo control franquista de las instituciones universitarias (De Moragas, 2013: 292), las perspectivas epistémicas que en origen delimitan las corrientes mayoritarias (Martínez Nicolás, 2006: 146) y la burbuja académica, alimentada, sobre todo, por la burbuja mediática (Díaz Nosty, 2011: 20 y 54).

[02] Las autoras utilizan el termino illuminating. En castellano hace referencia a algo esclarecedor o revelador. Aunque no se utilice normalmente el sustantivo esclarecimiento se ha optado por respetar la literalidad de la traducción en la cita reseñada.

[03] Según el Diccionario del Español actual (Seco, 1999) 'entelequia' puede definirse como «principio que determina el modo de existencia de un ser y hace que tienda por sí mismo a cumplirlo». 
Sobre la burbuja académica, pocas áreas de conocimiento han tenido un crecimiento tan acelerado desde su nacimiento. A mediados de los años ochenta del pasado siglo, cuatro facultades reunían unos 5.000 estudiantes. Diez años después había 20 facultades y 20.000 alumnos. En el año 2.000 eran ya 44 facultades y 3.000 profesores (Martínez, Saperas y Carrasco, 2019: 45). Las últimas estadísticas oficiales disponibles hablan de 23.804 matriculados por 3.419 egresados para el año académico 2015/16 ${ }^{4}$. Desde el punto de vista interno de la comunidad, la burbuja académica ha sido sostenida por una «defensa acrítica, gremialista, de nuestros estudios, apoyando de facto un crecimiento por multiplicación sin reformas de fondo» (De Moragas, en Díaz Nosty, 2011: 59).

Según los últimos estudios ${ }^{5}$, actualmente existe una consolidada masa crítica de aproximadamente 2.500 investigadores que desarrollan su actividad en 56 centros de estudios, entre públicos y privados. Apuntaba De Moragas ( $u t$ supra), que existe una ausencia de políticas de coordinación entre centros que propulsa un ambiente excesivamente competitivo a todos los niveles: macro, meso y micro. Es decir, facultades que compiten entre sí por atraer alumnos, departamentos por organizar doctorados y másteres, grupos de investigación por conseguir fondos. Es a nivel micro donde esa competencia se hace aún más patente, compitiendo los individuos por engrosar currículos. Sin embargo, y pese a que la masa crítica es muy extensa, hay una suerte de inflación, tanto de estudios (Martínez y Saperas, 2011: 104) como de artículos (Goyanes, 2015: 90). En este sentido,

[...] la gran masa crítica de docentes de Comunicación en España, superior a la de cualquier otro país europeo, no genera una producción científica proporcional a los recursos disponibles» (De Moragas, en Díaz Nosty, 2011: 61).

Los efectos descritos pueden verse potenciados si los ponemos en relación con la cultura científica hegemónica, caracterizada por la asimilación de

[04] Las últimas estadísticas oficiales disponibles en la web oficial del Ministerio de Ciencia, Innovación y Universidades, publicadas el 25/06/2019, se refieren al año académico 2015-16. Estos números de estudiantes matriculados y egresados se refieren a grados en Periodismo, Comunicación Audiovisual e Información y Documentación. Se han excluido los estudios en Publicidad y Relaciones Públicas, siguiendo la propia delimitación del Ministerio, de http://www.educacionyfp.gob.es/servicios-al-ciudadano/estadisticas/universitaria/estadisticas alumnado/desde-2015.htm

[05] Datos procedentes del proyecto de investigación «El sistema de investigación en España sobre prácticas sociales de Comunicación. Mapa de Proyectos, Grupos, Líneas, Objetos de estudio y Métodos», con referencia CSO2013-47933-C4 
la cultura de la citación (Wouter, 1999) y del impacto (Diez-Gutiérrez 2017: 164 y De la Herrán y Villena (2012: 288). Las agencias de evaluación, y en concreto la ANECA, han optado expresamente por orientar el desempeño de los investigadores hacia criterios de producción intensiva (ANECA, 2017), asimilando y reforzando esta cultura, y situándonos en un escenario que va mucho más allá del manido «efecto ANECA» (Soriano, 2008).

En el caso de la comunicación, este paradigma ha desembocado en «un contexto académico de altísima presión por publicar [...] orientado por estrictos criterios de rentabilidad curricular» (Martínez, Saperas y Carrasco, 2019: 65) que ha causado una sensación generalizada de fatiga y estrés por publicar motivada por la necesidad de «incrementar la reputación profesional y dejar huella en la especialidad» (Goyanes y Rodríguez, 2018: 555).

Interesa, por tanto, orientar este análisis hacia la praxis discursiva, basado en tres macrotemas que se han considerado estratégicos para el funcionamiento del SIEC: la producción, evaluación y la gestión de la investigación.

\section{Objetivos y método}

El objetivo de este estudio es analizar la percepción de la comunidad investigadora sobre el sistema de investigación y evaluación en comunicación. Con ello, se pretende ofrecer un marco de comprensión sobre la influencia del contexto académico y científico en la praxis discursiva del ecosistema académico en Comunicación. Esta investigación se ha construido desde una perspectiva cualitativa de naturaleza exploratoria.

No se pretende por tanto describir una realidad segmentada artificialmente, sino más bien interpretar una serie signos externos que hablan de un contexto determinado. La herramienta de análisis es una encuesta de actitud online. A pesar de que la técnica de encuesta se orienta tradicionalmente a la acumulación de descriptores cuantitativos, es también perfectamente válida para estructurar un análisis de naturaleza cualitativa. A la par que aporta datos estadísticos pormenorizados sobre las cuestiones planteadas, ofrece un panorama susceptible de ser estudiado desde un prisma cualitativo, como por ejemplo, opiniones, actitudes o diversidad de los temas tratados. Por lo tanto, este tipo de encuesta «establece la variación significativa (las dimensiones y valores relevantes) dentro de esa población» (Jansen, 2012: 41) y «no tiene en cuenta la frecuencia de las categorías (o valores), sino que busca la diversidad empírica de las propiedades de los miembros, incluso si estas propiedades se expresan en números» (Jansen, 2012: 45).

Efectivamente, para describir un determinado contexto, más que el dato estadístico en sí, o la moda que arroje, interesa más ver la diversidad 
de percepciones en torno a los tres grandes temas planteados en la encuesta: la producción, evaluación y gestión de la investigación. Estas percepciones serán ponderadas siguiendo el sistema Likert, que es una escala de valoración de actitud con amplia tradición en las ciencias sociales. Siguiendo a las expertas en psicología social Alice H. Eagly y Shelly Chaiken (1993: 1), la actitud puede ser definida como «una tendencia psicológica que se expresa al evaluar una entidad particular favorable o desfavorablemente». La actitud viene a reflejar un conjunto de elementos que influencian al individuo, entre los cuales se encuentra el contexto. En este sentido,

[...] estamos de acuerdo en que los efectos de contexto son generalizados. Los efectos del contexto, debido a la presencia de una audiencia u otras señales, son omnipresentes porque los juicios evaluativos no son expresiones puras de actitud, sino resultados que reflejan información en la situación actual, así como al menos algunos aspectos de la tendencia evaluativa preexistente [...] Además, aparte de las influencias derivadas de la situación externa, la relación entre la tendencia evaluativa misma y una expresión evaluativa dada no es uno a uno porque cada respuesta sólo mapea de manera imperfecta la evaluación (Eagly y Chaiken, 2007: 587).

Por lo tanto, de esta afirmación se derivan tres ideas fundamentales que han servido a este trabajo:

$\rightarrow 1$. El contexto genera una tendencia evaluativa.

$\rightarrow$ 2. Los juicios evaluativos no son expresiones puras de actitud.

$\rightarrow$ 3. La expresión de la actitud no se corresponde con la actitud misma y su análisis, desde una dimensión discursiva, nos aproxima más hacia el análisis del contexto que la realidad misma de la actitud.

La encuesta contempla veinte ítems, más dos de control que siguen la escala Likert. Se ha optado por el sistema de cinco categorías, por ser el que más consenso científico suscita para favorecer el incremento de respuestas debido a la facilidad con que se puede entender la escala (Dawes, 2008: 62). Estas cinco categorías son: totalmente de acuerdo, de acuerdo, indeciso, en desacuerdo y totalmente en desacuerdo. Los enunciados pretenden estimular el posicionamiento subjetivo del individuo, por lo que no eluden entrar en cuestiones claramente controvertidas. La clave es no dejar a ningún suje- 
to indiferente ante los enunciados y provocar una reacción de aprobación o desaprobación. Por lo tanto, no procede en este diseño metodológico una estructura basada en una pretendida falta de sesgo sino más bien en un enunciado con una clara tendencia ideológica, sea en sentido favorable o desfavorable. Esto va en consonancia con el enfoque epistemológico y también en coherencia con la finalidad del trabajo: visualizar la percepción dominante de la comunidad investigadora.

Los ítems se han distribuido en tres áreas esenciales del funcionamiento del SIEC: la producción, evaluación y gestión del conocimiento. Sobre cada uno de estos macrotemas se desarrollaron una serie de subtemas al objeto de extraer los ítems de forma proporcionada y representativa. Los enunciados planteados se encuentran numerados para facilitar su interpretación cruzada.

En relación a la producción de la investigación, las cuestiones planteadas inciden en aspectos sobre la forma y modos de investigar: estructura y forma de los outputs, elección de temas y líneas de investigación, canales productivos, innovación y estandarización del discurso científico. Respecto a la evaluación, las cuestiones se refieren a los criterios dominantes, asimilación del modelo nomotético en el discurso y hegemonía narrativa de las ciencias naturales de la evaluación científica. Finalmente, sobre el área de la gestión, se exponen temas sobre las condiciones de producción, funcionamiento de las instituciones y burocratización de la actividad.

Las encuesta se lanzó entre el 9 y el 25 de mayo del 2018 a un total de 2.418 investigadores en comunicación. En ese transcurso de tiempo se mandó un mensaje recordatorio el día 18. Para establecer el universo de investigadores en comunicación se utilizaron los datos recogidos en el marco del proyecto de investigación MapCom ${ }^{6}$, que estableció por primera vez en España un censo de 2.418 investigadores de la comunicación a partir de tres criterios:

$\rightarrow$ Doctores/as con relación laboral en las Facultades de Comunicación.

$\rightarrow$ Doctorandos/as que reciben la formación de las Facultades de Comunicación.

$\rightarrow$ Doctores/as sin relación laboral en las Facultades pero afiliados a alguna asociación científica.

[06] MAPCOM es el acrónimo del proyecto de investigación «El sistema de investigación en España sobre prácticas sociales de Comunicación. Mapa de Proyectos, Grupos, Líneas, Objetos de estudio y Métodos», con referencia CSO2013-47933-C4. Este proyecto se ejecutó desde el año 2014 hasta finales de 2017, de https://www.mapcom.es/home 
El número total de respuestas completas obtenidas es de 604, lo que supone una tasa de respuesta del 24,97 por ciento. Respecto al perfil de los encuestados, el 52,5 por ciento son hombres (317) y el 47,5 por ciento mujeres (287). Para completar el perfil se ha considerado necesario incluir una dimensión laboral. En este sentido, se han tenido en cuenta las diferentes escalas de acreditación oficial que actualmente se encuentran en vigor y se ha contrastado la información obtenida con la situación contractual real. La participación se ha concentrado en las escalas presumiblemente más estables: contratados doctores y titulares. La participación de 52 catedráticos/as de universidad aporta un valor añadido a esta encuesta, al ser una muestra muy representativa del escalón sénior de la investigación en comunicación en España.

Tabla 1. Perfil laboral.

\begin{tabular}{|l|r|r|}
\hline \multicolumn{1}{|c}{ Tengo la acreditación de... } & \multicolumn{2}{c|}{ Número de respuestas } \\
\hline No tengo ninguna ni se encuentra entre mis objetivos & 23 & 3,8 \\
\hline $\begin{array}{l}\text { No tengo ninguna pero estoy preparándome para PAD } \\
\text { (profesor/a ayudante doctor/a) }\end{array}$ & 67 & 11 \\
\hline PAD & 40 & 6,6 \\
\hline $\begin{array}{l}\text { Profesor/a Contratado/a Doctor/a o profesor/a de } \\
\text { Universidad Privada }\end{array}$ & 216 & 35,8 \\
\hline Titular de Universidad & 206 & 34,1 \\
\hline Catedrático/a de Universidad & 52 & 8,6 \\
\hline
\end{tabular}

Fuente: elaboración propia.

Además de las acreditaciones, la realidad contractual real puede aportar información muy valiosa sobre el perfil de los encuestados. En este sentido, se ha considerado necesario valorar si la posición por la que se está acreditado coincide plenamente o no con la realidad contractual de cada caso.

Tabla 2. Equivalencia entre acreditación y situación laboral.

\begin{tabular}{|l|r|r|}
\hline \multicolumn{1}{|c}{ Equivalencia } & \multicolumn{2}{c|}{ Número de respuestas } \\
\hline Coincide con la figura para la que está acreditado & 366 & 60,6 \\
\hline No coincide con la figura para la que está acreditado & 184 & 30,5 \\
\hline No procede & 54 & 8,9 \\
\hline
\end{tabular}

Fuente: elaboración propia.

Existe un alto porcentaje de sujetos que manifiestan una posición no equivalente $\mathrm{y}$, por lo tanto, no consolidada a nivel laboral respecto a los méritos curriculares reconocidos por la ANECA. Esta situación puede provocar disfunciones a muchos niveles en el individuo y en su relación con el entorno. No corresponde a este estudio entrar en esos campos. Sin embargo, es información de contexto muy relevante para crear una imagen sociolaboral actualizada. 


\section{Resultados y discusión}

La encuesta se ha organizado en tres temas considerados esenciales para el funcionamiento del SIEC: la producción, evaluación y gestión de la investigación. De cada de una de estas tres áreas se han desarrollado enunciados que exponen subtemas controvertidos. Estos se encuentran numerados para facilitar un análisis interpretativo cruzado. Se ha optado por presentar los resultados en porcentaje más que en número de respuestas. En este sentido, interesa de este análisis valorar la diversidad y las tendencias mayoritarias hacia una determinada posición del contexto más que establecer un valor estadístico medio o entrar en cuestiones aritméticas, por lo que no debe excluirse una interpretación conjunta entre los enunciados de los tres temas. Esa perspectiva no se corresponde con el enfoque epistemológico de este trabajo.

a) Percepción de la producción de la investigación. El área de la producción de la investigación capitaliza gran parte de la actividad de los investigadores, por lo que se ha visto oportuno desarrollarla de forma más extendida que el resto de áreas: 10 enunciados y 6 en el resto. La pregunta general es: ¿Hacia dónde se orienta la percepción sobre los modos de producción de la investigación? Ver gráfico 1.

Existe una clara tendencia a valorar positivamente los estudios de naturaleza cualitativa (e1). Sobre la percepción de la estandarización del discurso científico, la comunidad investigadora opina mayoritariamente, con un 60 por ciento de opiniones favorables, que el discurso actualmente está estandarizado (e2). Si ponemos en relación esta percepción con los rasgos de la evaluación que afectan de lleno a las formas y estilos de los outputs, se podría argumentar que esta estandarización está muy influenciada por los criterios evaluativos imperantes, que dificultan la innovación de la investigación. El 83 por ciento de los encuestados opina que «hay poco espacio para la innovación». Destaca la idea utilitaria del 42 por ciento de la opinión de elegir antes un tema publicable que otro de interés para el propio sujeto (e9), así como ver la hiperespecialización como una estrategia óptima para garantizar la supervivencia en el mundo académico (e10).

Tabla 3. Elijo antes un tema publicable que un tema que me interese más.

\begin{tabular}{|l|r|r|}
\multicolumn{2}{|c|}{ Escala } & Número respuestas \\
\hline Totalmente de acuerdo & 103 & 17,1 \\
\hline De acuerdo & 153 & 25,3 \\
\hline Indeciso/a & 55 & 9,1 \\
\hline En desacuerdo & 215 & 35,6 \\
\hline Totalmente en desacuerdo & 78 & 12,9 \\
\hline & 604 & 100,0 \\
\hline
\end{tabular}

Fuente: elaboración propia. 
Gráfico 1. Percepción sobre producción científica.

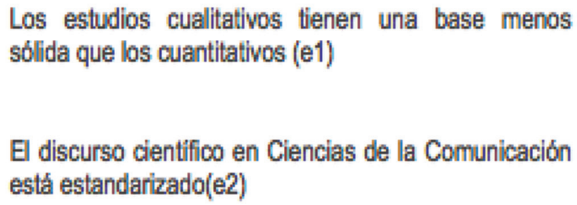

El discurso científico en Ciencias de la Comunicación está estandarizado(e2)

Hay poco espacio para la innovación con los actuales criterios evaluadores( $\mathrm{e} 3$ )

Lo más importante de una carrera investigadora es publicar en JCR/SJR(e4)

La metodología de una investigación es la parte más importante de su estructura(e5)

Al completar la estructura IMRAD la investigación en Comunicación pierde muchos matices(e6)

Cumplir con la narrativa predeterminada IMRAD (Introduction, Methodology, Results, Analysis and Discussion) supone un esfuerzo añadido para mi investigación social(e7)

Entre revistas, congresos y demás actividades, existe una proliferación excesiva de canales productivos que dispersan la actividad investigadora(e8)

Elijo antes un tema publicable que un tema que me interese más(e9)

La hiperespecialización es una forma de sobrevivir en el mundo académico(e10)
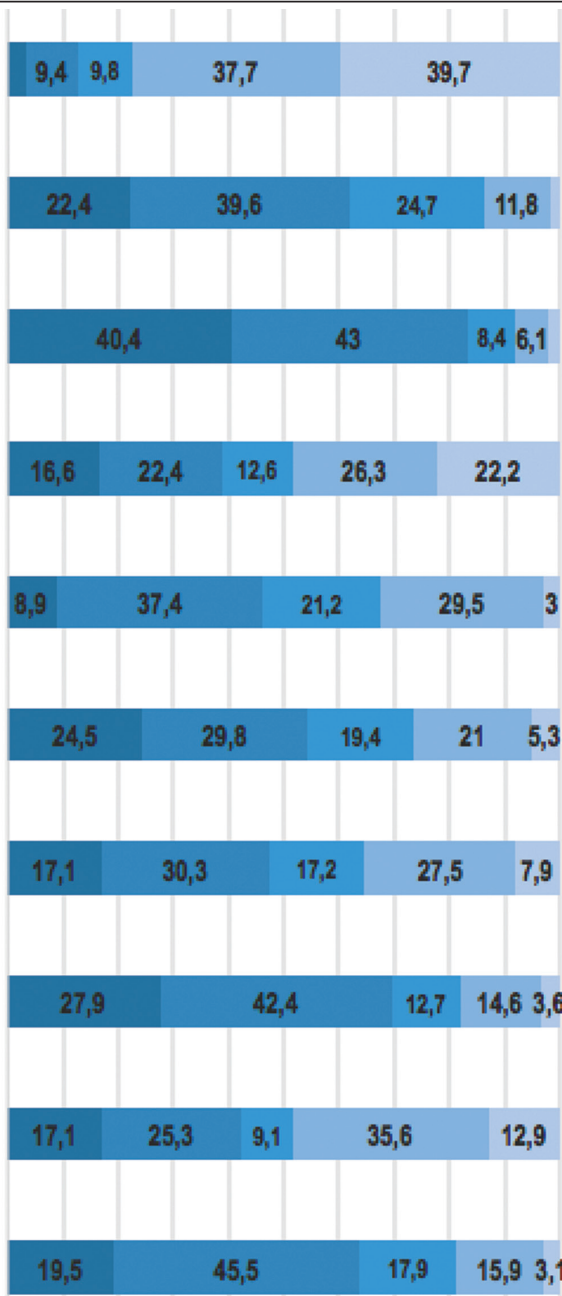

- Totalmente de acuerdo $\quad$ De acuerdo = Indeciso = En desacuerdo = Totalmente en desacuerdo

Fuente: elaboración propia.

El 39 por ciento de las personas encuestadas está de acuerdo en considerar que lo más importante de una carrera académica es publicar en los índices de impacto conocidos como JCR/SJR. De esos, el 16,6 por ciento, es decir, 100 personas, están totalmente de acuerdo en esa afirmación (e4). 
Sobre la estructura de las publicaciones científicas, la opinión predominante considera que al cumplir con la narrativa predeterminada $\operatorname{IMRAD}^{7}$ (introduction, methodology, results, analysis and discussion) se pierden muchos matices en la investigación (e6), mientras que casi la mitad ve esta estructura como un esfuerzo añadido a la investigación (e7).

b) Percepción sobre la evaluación de la investigación. La evaluación sobre la investigación se configura como una dimensión fundamental en la carrera investigadora, sobre todo debido a los criterios utilizados por la ANECA respecto a los sistemas de acreditación. En el marco teórico se han trazado ya las líneas desde las que se ha enfocado esta encuesta. Se asume que actualmente vivimos en la cultura de la cita (Wouters, 1999) y del impacto (De la Herrán y Villena, 2012). $\mathrm{Al}$ introducir este tema en la encuesta se pretende contribuir a la comprensión de este fenómeno, que se ha convertido en un eje de la praxis académica actual.

[07] A comienzos de los años setenta del pasado siglo se adoptó como forma estandarizada del discurso científico el formato IMRyC, introducción, metodología, resultados y conclusiones (del inglés IMRAD, introduction, methodology, results, analysis and discussion). Esta norma fue aprobada por el Instituto Nacional Estadounidense de Estándares en 1972 (ANSI Z39.16-1972), y nuevamente en 1979 (Wu, 2011: 1348). A partir de los años ochenta su implantación se va generalizando a todas las ramas científicas. El origen de fórmulas que estandarizan la narrativa científica puede deberse a dos presupuestos: por un lado, la imposición del paradigma de la replicación, como requisito indispensable de la ciencia (enfoque experimental contra enfoque hermenéutico). Y por otro, como forma de controlar el enorme flujo de artículos antes de la explosión de la comunicación científica que se produce a partir de la segunda mitad del siglo xx (Day, 1998: 6-11). 
Gráfico 2. Percepción sobre evaluación de la investigación.

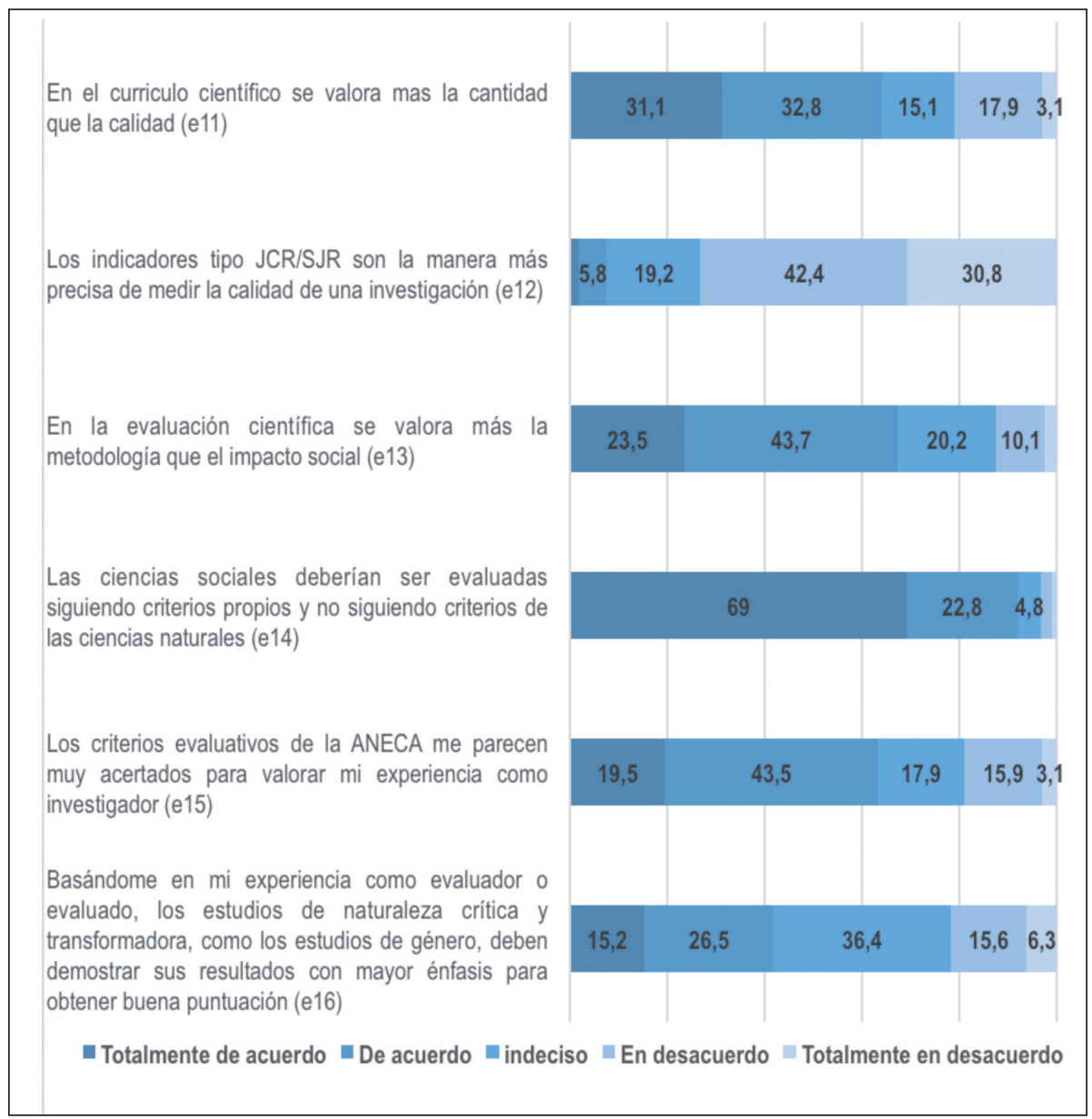

\section{Fuente: elaboración propia.}

Una mayoría del 73,2 por ciento no considera preciso los indicadores tipo JCR/SJR para medir la calidad de una investigación y, sin embargo, asimila los criterios evaluativos de la ANECA como muy acertados (63 por ciento) para valorar la experiencia como investigador (e15). 
Tabla 4. Indicadores JCR/SJR, el modo más preciso de medir la calidad de una investigación.

\begin{tabular}{|l|r|r|}
\multicolumn{2}{|c}{ Escala } & \multicolumn{2}{c|}{ Número respuestas } \\
\hline Totalmente de acuerdo & 11 & 1,8 \\
\hline De acuerdo & 35 & 5,8 \\
\hline Indecisola & 116 & 19,2 \\
\hline En desacuerdo & 256 & 42,4 \\
\hline Totalmente en desacuerdo & 186 & 30,8 \\
\hline & 604 & 100,0 \\
\hline
\end{tabular}

Fuente: elaboración propia.

Predomina la idea, con un 63,9 por ciento, de que en la evaluación del currículo científico se valora más la cantidad que la calidad (e11), destacando de este porcentaje que más de un tercio de todas las repuestas (188) ha manifestado estar «totalmente de acuerdo» con esta aseveración. Por otra parte, existe la percepción generalizada (92 por ciento) de que las ciencias sociales deberían ser evaluadas siguiendo criterios propios y no provenientes de las ciencias de la naturaleza (e14). Esta contundencia sugiere un replanteamiento de las prácticas. Es destacable que el 41,2 por ciento de la opiniones ha considerado que los estudios de naturaleza crítica tienen el deber de demostrar más y mejor los resultados de cara a las evaluaciones (e16).

c) Percepción sobre la gestión. La burocratización de la actividad investigadora es un hecho de la cultura científica actual. Saber en qué medida es importante para la comunidad investigadora y si afecta o no a la propia actividad es la finalidad de este apartado. Ver gráfico 3.

El 85,4 por ciento de las personas encuestadas manifiesta que «las tareas administrativas relacionadas con la evaluación afectan al diseño de futuras investigaciones» (e17), mientras que el 69,7 por ciento está totalmente de acuerdo en afirmar que emplea un tiempo excesivo en tareas administrativas. Estos números sugieren que la escasez de tiempo es percibido como un problema real que afecta en tiempo y forma a la propia investigación.

Respecto a la percepción de representatividad en los órganos de decisiones hay una desconexión clara y manifiesta entre el funcionamiento, diseño y aplicación de políticas científicas relativas a la evaluación y las opiniones predominantes. Ante la afirmación «la comunidad científica participa lo suficiente en el diseño de los criterios de evaluación científica» (e22), el 64,1 por ciento opina desfavorablemente, destacando el que el 20,2 por ciento se muestra «totalmente en desacuerdo». Asimismo el 77,9 por ciento asocia más participación con mejores resultados (e21). 
A nivel laboral, con un 93,1 por ciento de las respuestas, es decir, 562 personas perciben que sufren precariedad laboral ${ }^{8}$. La mayoría de los encuestados manifiesta el máximo grado de acuerdo con la afirmación contenida en el enunciado 20. En términos de valorar cómo aprecia el sujeto su puesto de trabajo y las condiciones en las que se desenvuelve, la percepción generalizada, con un 74,7 por ciento de las respuestas, es que habitan en un entorno basado en la competencia más que en la cooperación (e19).

\section{Gráfico 3. Percepción sobre gestión.}

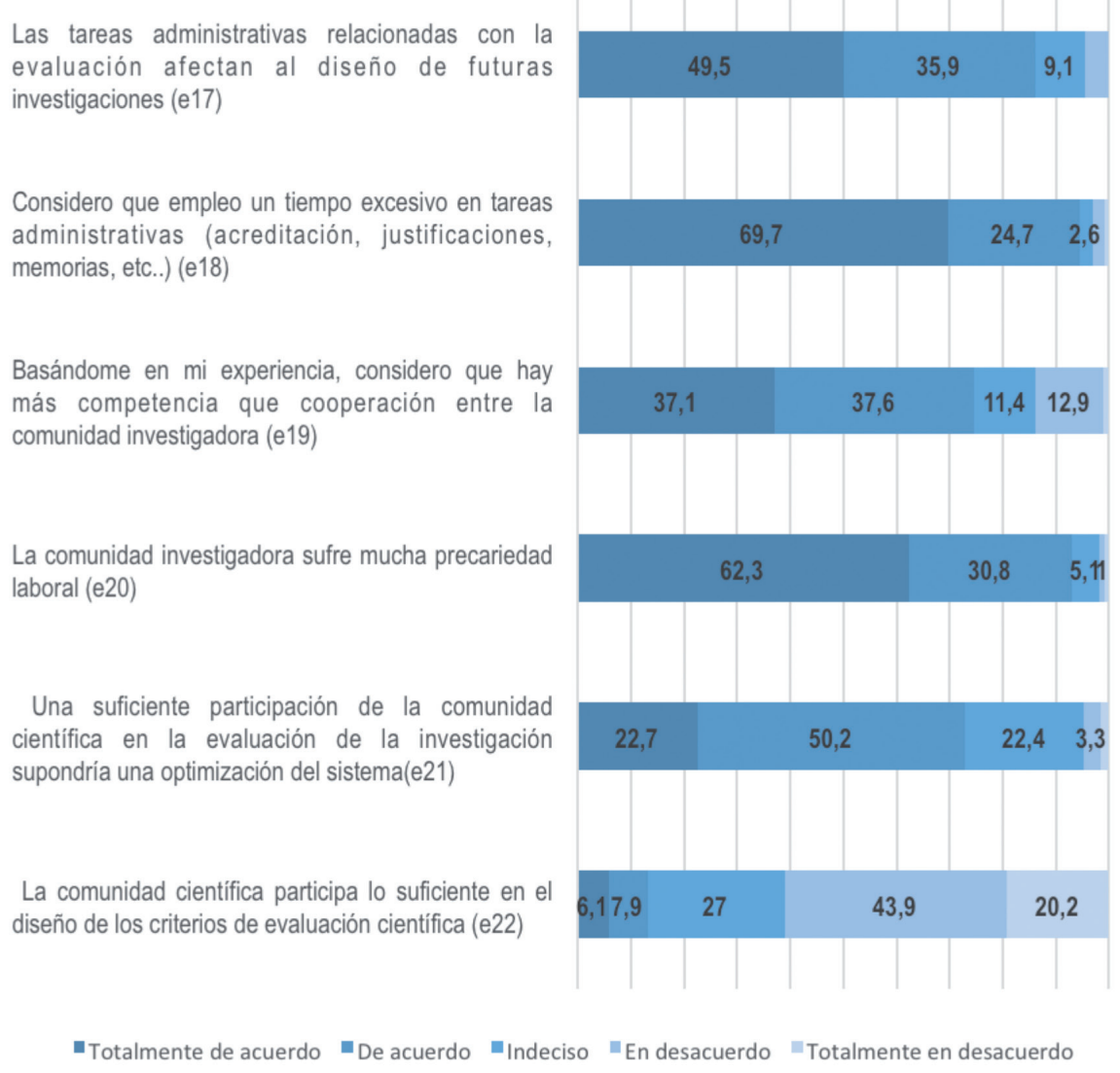

Fuente: elaboración propia.

[08] Actualmente, el autor de este artículo recibe 1.086,68 euros netos $-1.466,40$ euros brutos- como investigador a tiempo completo con titulación superior de la Universidad de Málaga. Estos datos arrojan una diferencia de 8.327,63 euros por debajo del salario medio nacional, según los últimos datos disponibles del INE para el año 2016. En https://www.ine. es/jaxiT3/Datos.htm?t=10882 
Tabla 5. Basándome en mi experiencia, considero que hay más competencia que cooperación entre la comunidad investigadora.

\begin{tabular}{|l|r|r|}
\hline \multicolumn{2}{|c}{ Escala } & \multicolumn{2}{c|}{ Número de respuestas } \\
\hline Totalmente de acuerdo & 224 & 37,1 \\
\hline De acuerdo & 227 & 37,6 \\
\hline Indeciso/a & 69 & 11,4 \\
\hline En desacuerdo & 78 & 12,9 \\
\hline Totalmente en desacuerdo & 6 & 1 \\
\hline & 604 & 100,0 \\
\hline
\end{tabular}

Fuente: elaboración propia.

\section{Conclusiones}

La combinación de los valores estudiados permite aventurar conclusiones que pueden servir para orientar futuros trabajos. En primer lugar, existe la percepción generalizada de que el discurso científico está estandarizado (e2) y de que se deben adoptar ciertas estrategias (e9, e10, e11, e16) para sobrevivir en un entorno de tanta competencia (e19). Estas estrategias hablan de potenciar la producción en serie de outputs científicos utilizando la diversidad de canales productivos al respecto (e8), centrándose en temas seguros y publicables (e9) o buscando fórmulas hiperespecializadas (e10).

Casi el 40 por ciento de las personas encuestadas percibe que asumir trabajos de naturaleza crítica y transformadora, o investigaciones basadas en enfoques de género, puede suponer un problema añadido (e16). Como resultado, los sujetos consideran mayoritariamente que el marco discursivo no favorece la innovación ni disrupción del discurso (e2, e3), así como que el espacio se encuentra totalmente acotado por los actuales criterios evaluadores (e3, e4, e12) y las formas hegemónicas provenientes de otras disciplinas científicas (e6, e7, e13, e14).

Sin embargo, es posible que los sujetos hayan asumido este modelo basado en la cultura de rendición de cuentas (e15), aunque no se identifiquen en absoluto con el funcionamiento de las instituciones evaluadoras (e21, e22). Una posible explicación de la asimilación de estos criterios puede ser leída en los términos de un entorno percibido como hostil ${ }^{9}$, donde impera la competencia más que la cooperación (e19,e20) y con un alto grado de burocratización, que es percibida como un obstáculo real (e17, e18).

Como debilidades de este análisis se observa la falta de desagregación de datos, que hubiera posibilitado un estudio por segmentos estratégicos, espe-

[09] En este punto se hace necesario acudir a la expresión «efecto termita del reparto de la miseria» del profesor Díaz Nosty cuando hablaba de la competencia dentro de la Universidad. Consultado en https://www.malagahoy.es/opinion/articulos/Chomsky_0_129587466.htm 
cialmente relevante para aplicar un enfoque de género y/o ver la diferencias intergeneracionales. No obstante, la principal fortaleza puede ser contribuir al análisis del contexto de la investigación en comunicación desde la praxis discursiva.

Como futuras líneas de investigación convendría explorar fórmulas que potencien la democratización de las instituciones que regulan la práctica científica, asegurando de esta manera una mayor representatividad de todos los colectivos y segmentos que conforman la comunidad investigadora.

\section{Bibliografía}

ANECA (2017): Méritos evaluables para la acreditación nacional para el acceso a los cuerpos docentes universitarios. Área de conocimiento: D. Ciencias Sociales y Jurídicas. Consultado en http://www.aneca.es/content/download/13782/171461 file/CRITERIOS_SOCIALES_JURIDICAS.pdf

Caffarel, C.; Domínguez, M. y Romano, V. (1989). El estado de la investigación en comunicación en España (1978-1987). Cuadernos de investigación en comunicación (C.i.n.c.o.), 3, 45-57.

CAffarel, C. y CÁCERes, D. (1993). La comunicación en España: planteamientos temáticos y metodológicos entre 1987 y 1990. La investigación en España. Cuadernos de investigación en comunicación (C.i.n.c.o), 29, 23-30. Consultado en http://www.quadernsdigitals.netddatos/hemeroteca/r_32/nr_447/a_6136/6136.pdi

Caffarel, C.; Ortega, F. y GaitÁn, J. A. (2017). Investigación en Comunicación en la Universidad Española en el periodo 2007-2014. El profesional de la información, 26(2), 218-227. Consultado en https://recyt.fecyt.es/index.php/EP//article, view/epi.2017.mar.08

CASTILlo, A. y CARRETón, M.C. (2010). Investigación en Comunicación. Estudio bibliométrico de las Revistas de Comunicación en España. Comunicación y Sociedad, 23(2), 289-327. Consultado en https://www.unav.edu/publicaciones/revistas index.php/communication-and-society/article/view/36234/30664

Costa-SÁnchez, C.(2017). Análisis de la productividad y visibilidad en Scopus de los investigadores españoles en Comunicación. OBS, 11(3), 1-16. Consultado en http://www.scielo.mec.pt/pdf/obs/v11n3/v11n3a01.pdi

Dawes, J. (2008). Do data characteristics change according to the number of scale points used? An experiment using 5-point, 7-point and 10-point scales. International Journal of Market Research, 50(1), 61-77. Consultado en http://citeseerx.ist.psu.edu/viewdoc/download?doi=10.1.1.417.9488\&rep=rep1\&type=pdi

DAY, R. (2005): Cómo escribir y publicar trabajos científicos, $3^{\mathrm{a}}$ ed. Washington, D.C.: The Oryx Press y Organización Panamericana de la Salud. 
De Filippo, D. (2013). La producción científica española en Comunicación en WOS. Las revistas indexadas en SSCI (2007-2012). Comunicar, 21(41), 25-34. Disponible en https://www.revistacomunicar.com/indice/articulo.php?numero=41-2013-02

De Moragas, M. (2011). Periodismo: formación desorientada. En Díaz Nosty, B. (Ed.) El libro negro del periodismo en España (pp. 59-61) Madrid: Cátedra UNESCO de Comunicación, Universidad de Málaga y Asociación de la Prensa de Madrid.

De Moragas, M. (2013). Interpretar la comunicación. Estudios sobre medios en América y Europa. Barcelona, España: Gedisa.

Delgado-López, E. y Repiso-Caballero, R. (2013). El impacto de las revistas de comunicación: comparando Google Scholar Metrics, Web of Science y Scopus. Comunicar, 21(41), 45-52. Disponible en https://www.revistacomunicar. com/indice/articulo.php?numero=41-2013-04

DENZIN, N. K. (1970). The research act: a theoretical introduction to sociological methods. London: Butterworths.

Denzin, N. K. y Lincoln, S. (2005). The sage handbook of qualitative research. (4. ${ }^{\mathrm{a}}$ ed.). London: Sage.

Díaz Nosty, B. (8 de marzo de 2008). Nuestro Chomsky. Malaga Hoy. Disponible en https://www.malagahoy.es/opinion/articulos/Chomsky_0_129587466.htm

Díaz Nosty, B. (2011). El libro negro del periodismo en España. Madrid, Cátedra UNESCO de Comunicación, Universidad de Málaga y Asociación de la Prensa de Madrid.

Díez-GutiérRez, J. E (2017). La cultura del impacto en la universidad española y la privatización del conocimiento público. Teknocultura. Revista de Cultura Digital y Movimientos Sociales, 14(1), 163-169. Disponible en https://revistas.ucm.es/index.php/TEKN/article/view/54095/51159

Eagly, A. H. y Chaiken, S. (1993). The psychology of attitudes. Orlando, FL, US: Harcourt Brace Jovanovich College Publishers.

Eagly, A. H., y Chaiken, S. (2007). The Advantages of an Inclusive Definition of Attitude. Social Cognition, 25(5) 582-602. Disponible en https://guilfordjournals. com/doi/pdf/10.1521/soco.2007.25.5.582

Gaitán, J. A.; Caffarel, C.; Lozano, C. y Piñuel, J. L. (2018). Condiciones y rendimientos de la Investigación en Comunicación: la visión de los académicos. CIC. Cuadernos de Información y Comunicación, 23, 105-123. Disponible en https://revistas.ucm.es/index.php/CIYC/article/view/60910/4564456547635

GoyANes, M. (2015). ¿Hacia una investigación estandarizada?. OBS 9(3), 85-99.

Disponible en http://obs.obercom.pt/index.php/obs/article/view/826

Goyanes, M. y Rodríguez-Gómez, E. F. (2018). ¿Por qué publicamos? Prevalencia, motivaciones y consecuencias de publicar o perecer. El profesional de la información, 27(3), 548-558. 
Herrán, A. y Villena, J. L. (2012). Algunas Críticas a la Evaluación del Profesorado Universitario Centrada en el Impacto. En A. DE LA Herrán y J. PAREDES (Coords.), Promover el cambio pedagógico en la universidad (pp. 305-336). Madrid: Pirámide.

Higgs, J.; DebBIE, H. y Grace, S. (2009). Writing qualitative research on practice. Rotterdam, Sense Publishers. Disponible en https://www.sensepublishers.com media/1291-writing-qualitative-research-on-practice.pdf

JANSEN, H. (2012). La lógica de la investigación por encuesta cualitativa y su posición en el campo de los métodos de investigación social. Paradigmas, 4, 39-72. Disponible en hittps://dialnet.unirioja.es/servlet/articulo?codigo=4531575

JoNES, Daniel E. (1998). Investigación sobre comunicación en España. Evolución y perspectivas. Zer Revista de estudios de comunicación, 5, 13-51.

KuHn, T. (1962). La estructura de las revoluciones cientificas. Chicago: University of Chicago Press.

MartíneZ-Nicolás, M. (2006). Masa (en situación) crítica. La investigación sobre periodismo en España: comunidad científica e intereses de conocimiento. Anàlisi, 33, 135-170. Disponible en https://www.raco.catindex.php/Analisi article/view/51745/56717

Martínez-Nicolás, M. y Saperas, E (2011). La investigación sobre Comunicación en España (1998-2007). Análisis de los artículos publicados en revistas científicas. Revista latina de Comunicación social, 66, 101-129. Disponible en http://www.revistalatinacs.org/11/art/926_Vicalvaro/05_Nicolas.htm|

Martínez-Nicolás, M.; SAPERAS, E. y CARRASCo-CAMPOS, A. (2019). La investigación sobre comunicación en España en los últimos 25 años (1990-2014). Objetos de estudio y métodos aplicados en los trabajos publicados en revistas españolas especializadas. Empiria. Revista de metodología de ciencias sociales, 42, 37-69. Disponible en http://revistas.uned.es/index.php/empiria/article/view/2325

Morin, E. (1994). Introducción al pensamiento complejo. Barcelona: Editorial Gedisa.

Peñafiel, C.; Torres, E. e IzQuierdo, P., (2017). Percepción cualitativa de gestores universitarios de investigación en Comunicación a través de un Philips 66. En Herrero, F. J. y Mateos, C. (Coord.). Del verbo al bit (pp. 790-810). Universidad de la Laguna. Disponible en http://www.revistalatinacs.org/16SLCS/librocolectivo-edicion-2.htm

PiñUel, J. L. y Morales, E. (2018). Un estudio praxeológico de la investigación en comunicación, a través del análisis del discurso hegemónico de investigadores académicos en España. Perspectivas de la Comunicación, 11(2), 83-135. Disponible en http://www.perspectivasdelacomunicacion.c.

Seco, M.; Andrés, O. y Ramos, G. (1999). Diccionario del español actual. Madrid, España: Aguilar. 
Soriano, J. (2008). El efecto Aneca. Investigar la comunicación. Asociación Española de Investigación de la Comunicación. Santiago de Compostela, 30 de enero a 1 de febrero, 2008.

VAn Disk, T. A. (2001). Algunos principios de una teoría del contexto. ALED, Revista latinoamericana de estudios del discurso, 1(1), 69-81. Disponible en http://discursos.org/oldarticles/Algunos\%20principios\%20de\%20una\%20teor\%EDa\%20del\%20 contexto.pdff

Vidal Beneyto, J. (1972). Las ciencias de la comunicación en la Universidad española. Bilbao: Zero S. A. Disponible en http://roderic.uv.es/bitstream handle/10550/50289/JVB23.pdf?sequence=18isAllowed=y

WitTGENSTEIN, L. (1953). Investigaciones filosóficas. Barcelona: Critica.

Wouters, P. (1999). The Citation Culture, Tesis doctoral. Universidad de Amsterdam. Disponible en http://garfield.library.upenn.edu/wouters/wouters.pdif

Wu, J. (2011). Improving the writing of research papers: IMRAD and beyond. Lanscape Ecol, 26, 1345-1349. Disponible en https:/llink.springer.com/content pdf/10.1007\%2Fs10980-011-9674-3.pdf

Para citar este artículo: Martínez García, R. (2019). La percepción de la comunidad académica sobre el sistema de investigación y evaluación en Comunicación. index.comunicación, 9(1), 197-216. 